\title{
Subsecond EEG-fMRI analysis for presurgical evaluation in focal epilepsy
}

\author{
Yuji Ito, MD, PhD,,-3 Satoshi Maesawa, MD, PhD, 1,4 Epifanio Bagarinao, PhD, ${ }^{1}$ Yu Okai, MD,,1,2 \\ Daisuke Nakatsubo, MD, ${ }^{1,4}$ Hiroyuki Yamamoto, MD, PhD, ${ }^{1,2}$ Hiroyuki Kidokoro, MD, PhD, ${ }^{1,2}$ \\ Naotaka Usui, MD, PhD, ${ }^{5}$ Jun Natsume, MD, PhD, ${ }^{1,2,6}$ Minoru Hoshiyama, MD, PhD, ${ }^{1}$ \\ Toshihiko Wakabayashi, MD, PhD, ${ }^{4}$ Gen Sobue, MD, PhD, ${ }^{1,7}$ and Norio Ozaki, MD, PhD ${ }^{1,8}$
}

${ }^{1}$ Brain \& Mind Research Center, Nagoya University, Nagoya, Aichi; Departments of ${ }^{2}$ Pediatrics, ${ }^{4}$ Neurosurgery, ${ }^{6}$ Developmental Disability Medicine, ${ }^{7}$ Neurology, and ${ }^{8}$ Psychiatry, Nagoya University Graduate School of Medicine, Nagoya, Aichi; ${ }^{3}$ Department of Pediatrics, Aichi Prefecture Mikawa Aoitori Medical and Rehabilitation Center for Developmental Disabilities, Okazaki, Aichi; and ${ }^{5}$ Department of Neurosurgery, National Epilepsy Center, Shizuoka Institute of Epilepsy and Neurological Disorders, Shizuoka, Japan

OBJECTIVE The authors recently reported a novel subsecond analysis method of analyzing EEG-functional MRI (fMRI) to improve the detection rate of epileptic focus. This study aims to validate the utility of this method for presurgical evaluation in pharmacoresistant focal epilepsy.

METHODS Among 13 patients with focal epilepsy undergoing presurgical examinations including simultaneous EEGfMRI at 3T, 11 patients had interictal epileptiform discharges (IEDs) during fMRI. The authors used the sequence of topographic maps during the IEDs as a reference to obtain subsecond fMRI activation maps with the same temporal resolution as the EEG data, and constructed "spike-and-slow-wave-activation-summary" (SSWAS) maps that showed the activation frequency of voxels during IEDs. Clusters were defined by thresholding the SSWAS maps (voxel value > 10 ), and those containing voxels with the top 3 highest activation frequencies were considered significant. Significant hemodynamic responses using conventional event-related (ER) analysis and SSWAS maps were compared with the resection areas and surgical outcomes at 1 year after surgery.

RESULTS Using ER analysis, 4 (36\%) of 11 patients had significant hemodynamic responses. One of 4 patients had significant hemodynamic responses in the resection area and good surgical outcome. Using SSWAS maps, 10 (91\%) of 11 patients had significant hemodynamic responses. Six of 10 patients had significant hemodynamic responses in the resection area, and 5 of the 6 patients achieved good surgical outcomes. The remaining 4 patients had significant hemodynamic responses distant from the resection area, and only 1 of the 4 patients achieved good surgical outcomes. The sensitivity, specificity, positive predictive value, and negative predictive value of SSWAS maps were 83.3\%, 75.0\%, $83.3 \%$, and $75.0 \%$, respectively.

CONCLUSIONS This study demonstrated the clinical utility of SSWAS maps for presurgical evaluation of pharmacoresistant focal epilepsy. The findings indicated that subsecond EEG-fMRI analysis may help surgeons choose the resection areas that could lead to good surgical outcomes.

https://thejns.org/doi/abs/10.3171/2020.1.JNS192567

KEYWORDS electroencephalography; epilepsy; functional magnetic resonance imaging; preoperative evaluation; simultaneous EEG-fMRI

$\mathrm{P}$ HARMACORESISTANT epilepsy accounts for approximately $30 \%$ of epilepsy cases..$^{27,32}$ This causes various comorbidities and diminishes quality of life in patients. In patients with intractable focal epilepsy, accu- rate identification of the epileptogenic network and eloquent cortices is essential for curative surgery. ${ }^{9,24}$ Although intracranial EEG (icEEG) recordings are usually required for presurgical evaluation, icEEG is invasive and can only

ABBREVIATIONS AED = antiepileptic drug; BOLD = blood oxygen level-dependent; ER = event-related; fMRI = functional MRI; FOV = field of view; FWEc = family-wise error cluster level correction; FWEp = family-wise error peak level correction; GLM = general linear model; HRF = hemodynamic response function; icEEG = intracranial EEG; IED = interictal epileptiform discharge; ILAE = International League Against Epilepsy; MNI = Montreal Neurological Institute; SOZ = seizure onset zone; SSWAS = spike-and-slow-wave-activation-summary; TLE = temporal lobe epilepsy.

SUBMITTED September 17, 2019. ACCEPTED January 7, 2020.

INCLUDE WHEN CITING Published online March 13, 2020; DOI: 10.3171/2020.1.JNS192567. 
cover a limited space..$^{15}$ Noninvasive multimodal evaluation prior to the placement of icEEG electrodes is needed for good surgical outcomes.

Recently, new noninvasive neurophysiological modalities have been advanced to detect the epileptic foci before implantation of icEEG electrodes. Simultaneous EEG-functional MRI (EEG-fMRI) is one of these modalities, and can identify brain regions associated with interictal epileptiform discharges (IEDs) by measurement of hemodynamic changes in blood oxygen level-dependent (BOLD) signals. ${ }^{11,12,22}$ Several studies have shown the clinical utility of EEG-fMRI for presurgical evaluation and demonstrated its high sensitivity and specificity using surgical outcomes as the reference standard. ${ }^{28,29}$ However, detecting IEDs on EEG recordings during fMRI is difficult in some patients due to the influence of the strong magnetic field inside the MRI machine. ${ }^{11}$ In other patients, detecting significant spike-related BOLD changes is challenging despite proper detection of IEDs. ${ }^{1,25}$

In our recent work, we proposed a new method of analyzing EEG-fMRI using the sequence of topographic maps during IEDs as a reference to detect subsecond fMRI activation changes. The obtained sequence of activation maps was then used to construct a "spike-andslow-wave-activation-summary" (SSWAS) map, which represented the number of times (frequency) a given voxel became active during the sequence. ${ }^{5}$ Although we have demonstrated the efficacy of using SSWAS maps to detect epileptic foci, we have not yet validated their usefulness for preoperative workup.

This study aimed to evaluate the utility of using SSWAS maps for presurgical evaluation, paying particular attention to the associations among the localization of spike-related BOLD changes observed in SSWAS maps, resection areas, and postoperative outcomes.

\section{Methods \\ Study Population}

Between January 2016 and June 2018, a total of 19 patients with pharmacoresistant focal epilepsy underwent radical resective surgery with at least 12 months of followup at Nagoya University Hospital. Among these 19 patients, 13 consented to participate in this prospective study and underwent EEG-fMRI recordings before surgery. Of the 13 patients, 11 displayed identifiable IEDs during simultaneous EEG-fMRI and were eligible for subsequent analysis. The position of icEEG placement and areas of resection were determined based on clinical data, scalp EEG findings, and neuroimaging modalities other than EEG-fMRI, with the EEG-fMRI results only used for method validation at this time.

This study was approved by the research ethics board of Nagoya University Graduate School of Medicine. Written informed consent was obtained from all patients or their parents before participation in this study.

\section{Acquisition of EEG-fMRI}

MR images were acquired using a Magnetom Verio 3-T scanner (Siemens) with a 32-channel head coil at the Brain and Mind Research Center of Nagoya University.
High-resolution T1-weighted images were acquired using a 3D magnetization prepared rapid acquisition gradient echo acquisition sequence: TR 2.5 seconds, TE 2.48 msec, 192 sagittal slices with a distance factor of $50 \%$ and 1 -mm thickness, in-plane voxel resolution $1 \mathrm{~mm} \times 1 \mathrm{~mm}$, field of view (FOV) $256 \mathrm{~mm} \times 256 \mathrm{~mm}$, and matrix size $256 \times 256$. All fMRI was recorded in a single continuous 15-minute session with a T2-weighted gradient echo planar imaging sequence: TR 2.5 seconds, TE $30 \mathrm{msec}$, FOV $192 \mathrm{~mm}$, matrix size $64 \times 64,39$ transverse slices with a $0.5-\mathrm{mm}$ interslice interval and 3-mm thickness, flip angle $80^{\circ}$, and a total of 360 volumes.

EEG and electrocardiogram data were simultaneously recorded inside the MRI scanner using a 32-channel MRI-compatible system (Electrical Geodesics Inc.). An EEG cap connected to a combined digitizer-amplifier system was worn by the patient throughout the scan and data from the amplifier sampled at $1 \mathrm{kHz}$ were continuously retrieved using NetStation software (version 5.0, Electrical Geodesics Inc.). ${ }^{20}$ Adult patients were instructed to keep their eyes closed and remain relaxed during the scan. Pediatric patients were sedated in a drug-induced sleep condition using triclofos sodium at $80 \mathrm{mg} / \mathrm{kg}$ body weight.

\section{Preprocessing for fMRI and EEG}

Data for fMRI were preprocessed using SPM software (version 12, Wellcome Trust Centre for Neuroimaging) running on MATLAB (MathWorks). The first 5 images were discarded to remove inhomogeneity at the beginning of the scan. The remaining images were corrected for temporal differences in slice acquisition and head motion, spatially normalized to the Montreal Neurological Institute (MNI) space, and smoothed with a Gaussian kernel of $8 \mathrm{~mm}$ full-width at half-maximum.

EEG data were preprocessed using NetStation software (version 5.0). The gradient artifact was removed using the template subtraction method, and the ballistocardiogram artifact was removed using principal component analysis. ${ }^{3,21}$ Two experienced epileptologists (Y.I. and S.M.) marked the onset of IEDs $\left(t_{\mathrm{on}}\right)$, which are similar to those recorded outside the MRI scanner (MRI preparation room) using the same instruments for 15 minutes just before the scan.

\section{Conventional Event-Related Analysis}

EEG-fMRI data were analyzed using SPM statistical software (version 12). Identified IED onsets were used to generate a series of spikes, which were then convolved with the canonical hemodynamic response function (HRF) and used in the statistical analysis of the preprocessed fMRI data using a general linear model (GLM) approach. ${ }^{2}$ For each individual, 1-tailed t-tests were applied to test IED-related regional BOLD signal changes. The significance level was set at $\mathrm{p}<0.05$ corrected for multiple comparisons using family-wise error cluster level correction (FWEc) with a cluster-forming threshold of $\mathrm{p}$ $=0.001$. When cluster size was more than 10,000 voxels, the significance level was reset to a more stringent threshold at $\mathrm{p}<0.05$ corrected for multiple comparisons using family-wise error peak level correction (FWEp) to further 


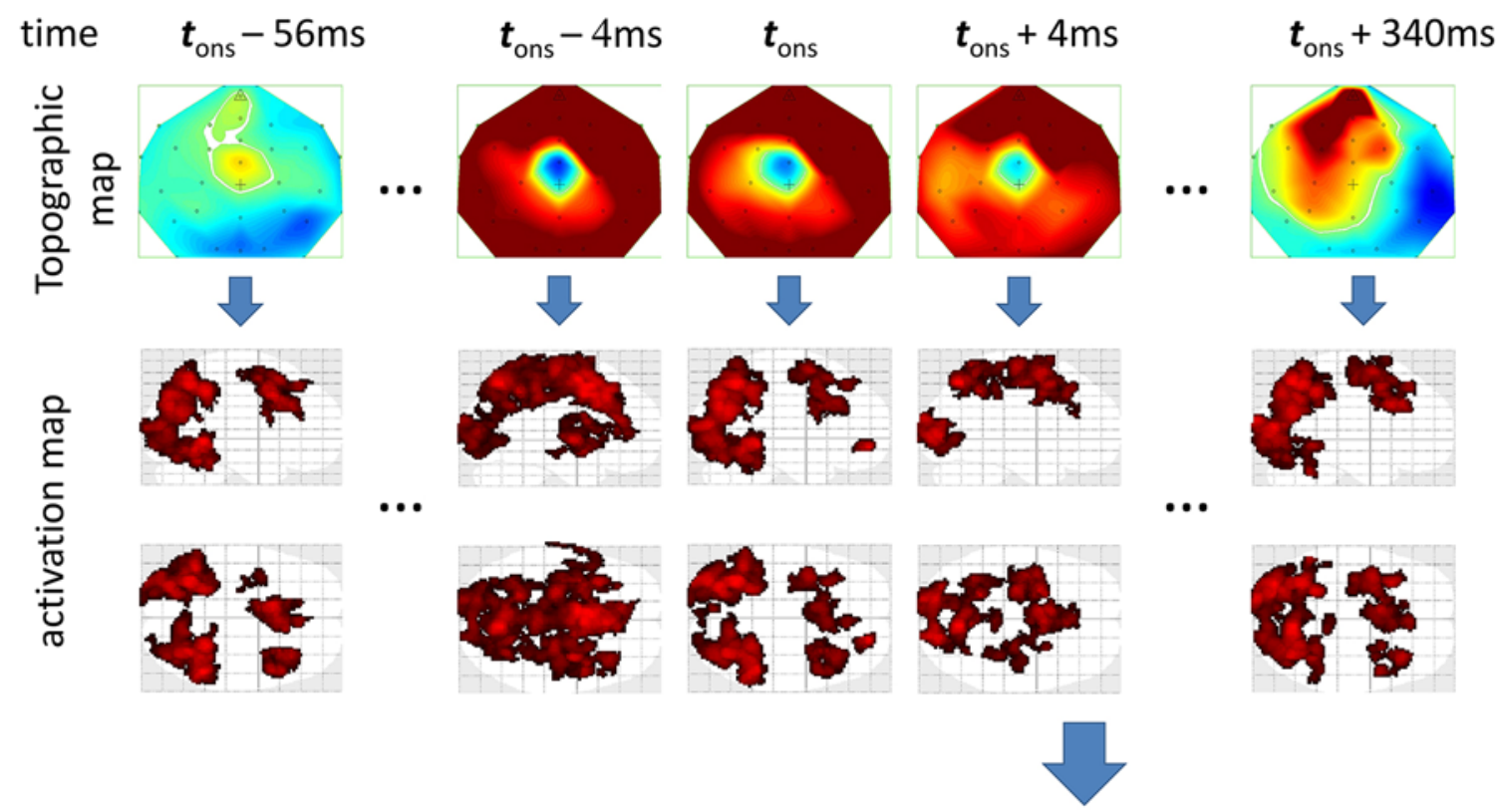

\section{SSWAS map \\ (spike-and-slow-wave-activation -summary map)}

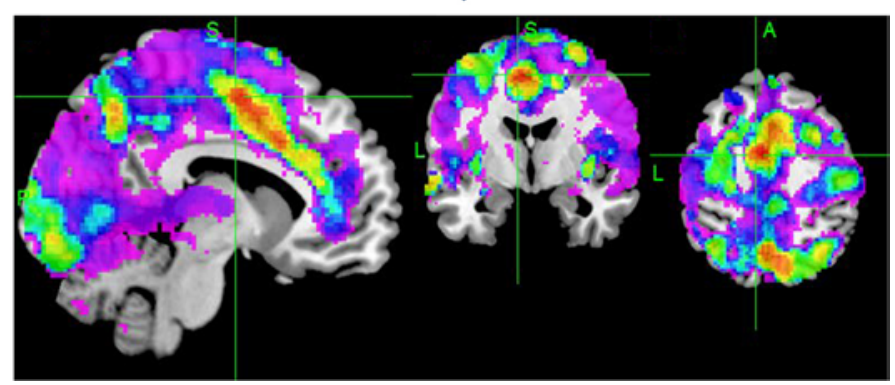

FIG. 1. Constructing SSWAS maps. The EEG topographic map at the time of onset of IEDs $\left(t_{\text {ons }}\right)$ was used as the reference map, and the similarity between the reference map and topographic map at any time $t$ was measured. The value of the similarity measure ranges from 0 to 1 , with 0 indicating no similarity and 1 being the same map. The time series of these similarity values were then convolved with the canonical HRF, and statistical analysis was performed using the GLM. The significance level was set at $p<0.05$ corrected for multiple comparisons using an FWEc. This process was repeated every $4 \mathrm{msec}$ (ms) in the sequence of topographic maps during the IEDs (from $t_{\text {ons }}-56 \mathrm{msec}$ to $t_{\text {ons }}+340 \mathrm{msec}$ ) to obtain subsecond fMRI activation maps with the same temporal resolution as the EEG data. From the sequence of activation maps, the SSWAS map, which showed the activation frequency (values ranging from 0 to 100) of voxels during the IEDs, was constructed. Figure is available in color online only.

identify significant peak locations within the cluster. We defined clusters containing voxels with the top 3 highest t-values as relevant to the epileptic foci. Activation maps were superimposed onto individual coregistered T1weighted images.

\section{Subsecond Analysis and Constructing SSWAS Maps}

The method used to analyze EEG-fMRI data to generate subsecond fMRI activation maps and to construct SSWAS maps was previously reported in more detail. ${ }^{5}$ This analysis was performed using an in-house MATLAB script and SPM software.

Basically, our approach advanced the method proposed by Grouiller and colleagues. ${ }^{13}$ Unlike conventional eventrelated (ER) analysis, this new method required wholescalp EEG topographic maps from simultaneously recorded scalp EEG data. The EEG topographic map at the time of $t_{\text {ons }}$ was used as the reference map, and the similarity between the reference map and the topographic map at any time $t$ was measured. The value of the similarity measure ranges from 0 to 1 , with 0 indicating no similarity and 1 being the same map. The time series of these similarity values were then convolved with the canonical HRF and used in the statistical analysis of the preprocessed fMRI data using the GLM approach. For each individual, 1-tailed t-tests were applied to test IED-related regional BOLD signal changes. The significance level of the obtained activation maps was set at FWEc $\mathrm{p}<0.05$.

This process was then repeated every $4 \mathrm{msec}$ in the sequence of topographic maps during the IEDs (from $t_{\text {ons }}$ $-56 \mathrm{msec}$ to $t_{\text {ons }}+340 \mathrm{msec}$ ) to obtain subsecond fMRI activation maps with the same temporal resolution as the EEG data. Next, the obtained sequence of activation maps was used to construct the SSWAS map, which showed the activation frequency (values ranging from 0 to 100) of voxels during the IEDs (Fig. 1). Clusters containing voxels with the top 3 highest activation frequency values and with voxel values greater than 10 were considered sig- 
nificant. SSWAS maps were superimposed onto the individual coregistered T1-weighted images.

\section{Comparison of BOLD Responses With Resection Areas and Surgical Outcomes}

Areas identified with IED-related hemodynamic responses using conventional ER analysis and the SSWAS map were compared using the resection areas and surgical outcomes at 1 year after surgery.

We judged agreement between the identified and resection areas as follows: 1) "concordant," when voxels having the first- to the third-highest t-values (for conventional ER analysis) or activation frequencies (for SSWAS maps) were inside the resection areas; 2) "borderline," when voxels were outside the resection areas, but $\leq 2 \mathrm{~cm}$ from the resection margin; and 3) "discordant," when voxels were distant from resection areas, $>2 \mathrm{~cm}$ from the resection margin.

Postoperative outcome was evaluated 1 year after surgery using the International League Against Epilepsy (ILAE) outcome classification scale. ${ }^{33}$ Briefly, ILAE class 1 means completely seizure free, class 2 means only auras, class 3 means 1-3 seizure days per year, class 4 means 4 seizure days per year to a $50 \%$ reduction in baseline seizure days, class 5 means a less than $50 \%$ reduction in baseline seizure days to a $100 \%$ increase in baseline seizure days, and class 6 means a more than 100\% increase of baseline seizure days. ILAE classes 1-2 were regarded as good surgical outcomes and classes 3-6 were regarded as poor surgical outcomes. We excluded "borderline" patients to calculate the sensitivity, specificity, positive predictive value, and negative predictive value, defined as follows: 1) sensitivity = patients with "concordant" and good outcome/patients with good outcome $\times 100 \%$; 2) specificity = patients with "discordant" and poor outcome/patients with poor outcome $\times 100 \%$; 3) positive predictive value = patients with "concordant" and good outcome/patients with "concordant" outcome $\times 100 \%$; and 4) negative predictive value $=$ patients with "discordant" and poor outcome/patients with "discordant" outcome $\times 100 \%$.

\section{Results}

The clinical characteristics of patients with focal epilepsy are shown in Table 1. Seizure types in all patients were only focal seizures. The median age at the time of imaging was 22 years, and 7 of 11 patients showed structural abnormalities on MRI. The two pediatric patients (patients 1 and 11) were sedated in the drug-induced sleep condition during EEG-fMRI. Patient 9 displayed two types of IED (frequent right parietal sharps and sporadic right temporal sharps), but only the right parietal sharp IEDs showed significant BOLD responses; therefore, these IEDs were the ones analyzed. Other patients exhibited only one type of IED. No patients experienced seizures during scans. All patients took more than two antiepileptic drugs (AEDs) before surgery. Patient 1 discontinued 3 AEDs completely within 1 year after surgery based on the wishes of the patient's parents. The number of AEDs was not reduced in other patients at 1 year after surgery. No patients showed postoperative motor, sensory, or cognitive deficits.
Using conventional ER analysis, 4 (36\%) of 11 patients had significant hemodynamic responses (Table 2). Patient 3 had both positive and negative BOLD responses, while the other 3 patients showed only positive BOLD responses. Because the primary cluster size was 28,412 voxels in patient 2, the level of significance was set at FWEp p < 0.05 for this patient. For all other patients, the significance level was set at FWEc $\mathrm{p}<0.05$. Patient 1 showed "concordant" BOLD responses and achieved good surgical outcomes. Patient 2 had "borderline" BOLD responses, but the BOLD cluster with the third-highest $t$-value $(\mathrm{t}=$ 5.97) overlapped the resection areas, and she too achieved good surgical outcomes (Fig. 2A). Patient 3 had "discordant" BOLD responses, but the BOLD cluster with the sixth-highest $t$-value $(t=5.19)$ was inside the resection area, and she experienced good surgical outcomes. Patient 7 had "discordant" BOLD responses and poor surgical outcomes. Other patients, including patient 5, showed no significant hemodynamic responses (Fig. 3A). The sensitivity, specificity, positive predictive value, and negative predictive value of conventional ER analysis were $50.0 \%$, $100.0 \%, 100.0 \%$, and $50.0 \%$, respectively.

Using SSWAS maps, 10 (91\%) of 11 patients had significant hemodynamic responses (Table 2). All 10 patients displayed positive BOLD responses only. Six of the $10 \mathrm{pa}-$ tients had "concordant" BOLD responses, and 5 of those 6 patients achieved good surgical outcomes (Figs. 2B and 3B). Widespread positive BOLD changes in patient 6 were observed bilaterally (right hemisphere dominant), and she showed poor surgical outcomes. The remaining 4 patients had significant hemodynamic responses distant from the resection area, and 3 of those 4 patients attained poor surgical outcomes. The sensitivity, specificity, positive predictive value, and negative predictive value of SSWAS maps were $83.3 \%, 75.0 \%, 83.3 \%$, and $75.0 \%$, respectively.

\section{Discussion}

In this prospective study of a tertiary epilepsy center cohort, we demonstrated the clinical utility of SSWAS maps for presurgical evaluation in pharmacoresistant focal epilepsy. Subsecond analysis can detect significant hemodynamic responses in approximately $90 \%$ of patients, while conventional ER analysis can only detect responses in one-third of patients. In the comparison of significant BOLD responses with the resection areas and surgical outcomes, SSWAS maps showed adequate sensitivity and specificity for presurgical evaluation. This suggests that subsecond EEG-fMRI analysis would be helpful to surgeons in identifying the optimal placement of icEEG electrodes to achieve good surgical outcomes.

SSWAS maps were able to detect the significant BOLD changes of epileptic foci where conventional ER analysis failed. EEG recordings inside the MRI scanner contain many artifacts, including those due to gradient and ballistocardiography. Although improvements in artifact removal techniques are reducing the difficulty of detecting IEDs, some IEDs are still easily missed, which affects the accuracy of statistical analysis..$^{10,31}$ Grouiller and colleagues showed that use of the reference topographic map on scalp EEG outside the scanner could help improve the 


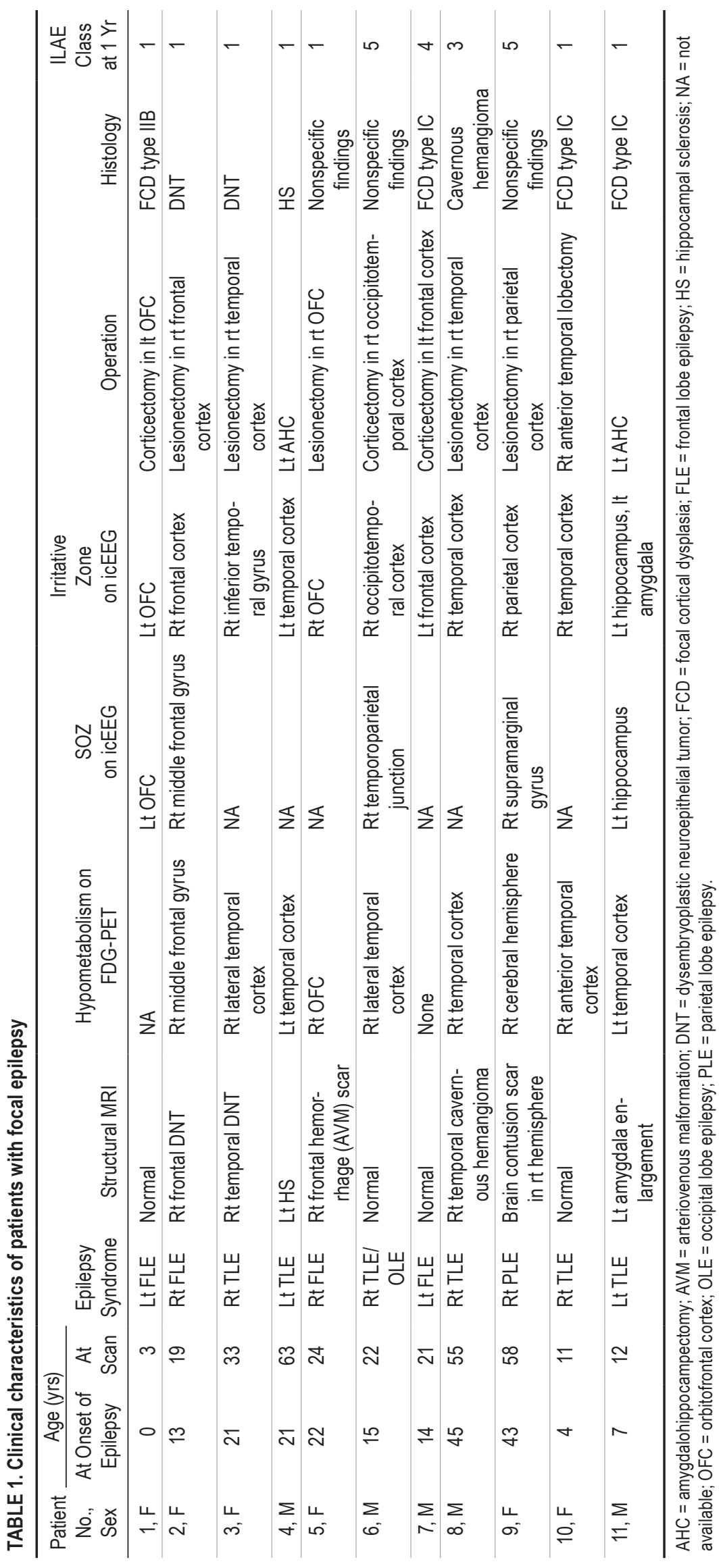




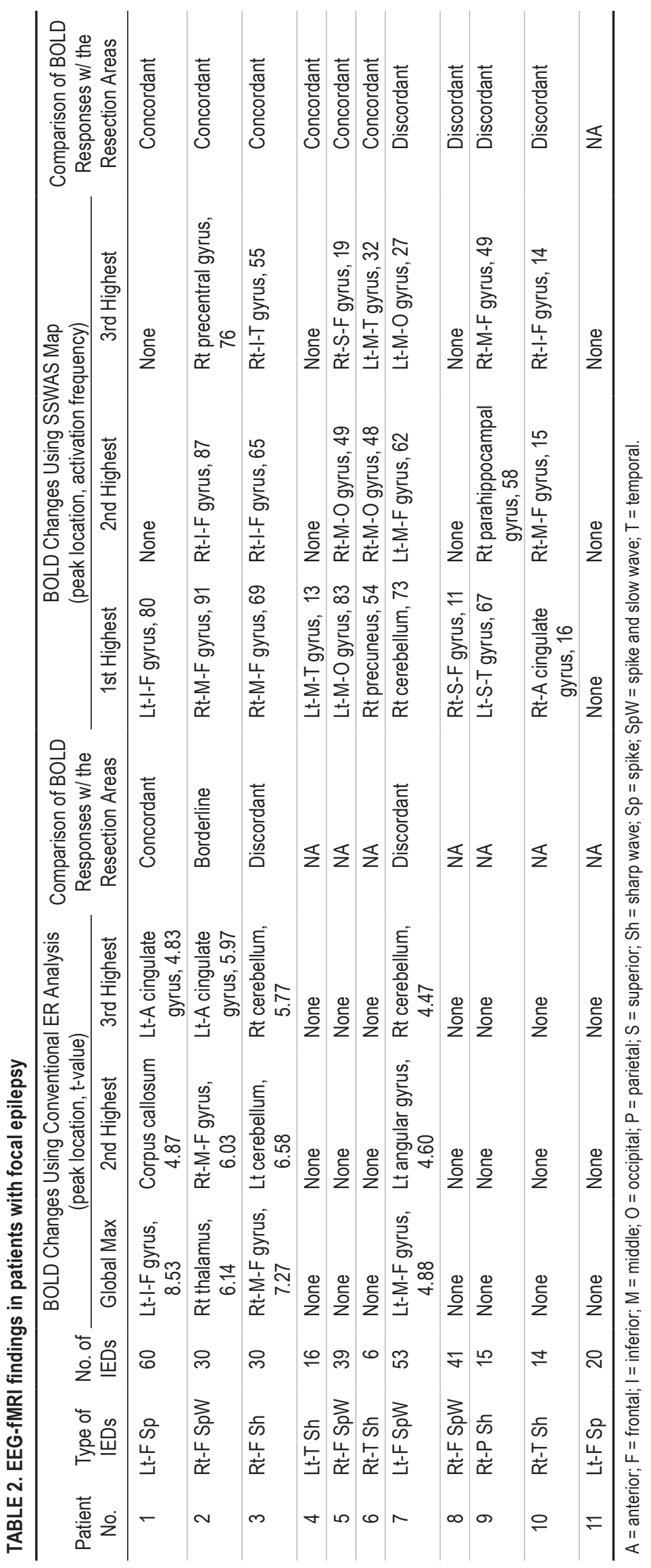




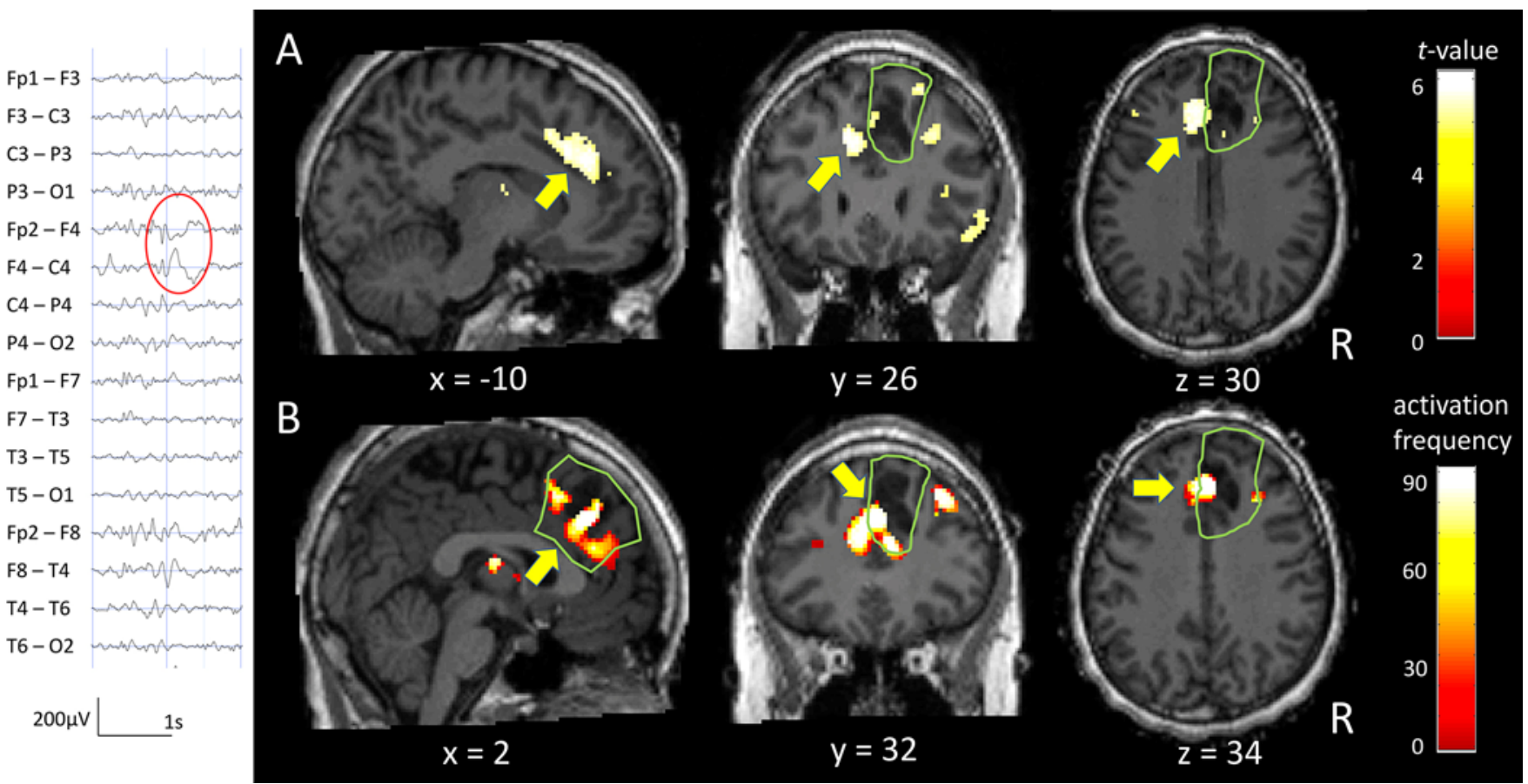

FIG. 2. Case 2. A case with nonconcordant hemodynamic responses using conventional ER analysis and concordant hemodynamic responses using subsecond analysis. This patient was a 19-year-old woman with right frontal lobe epilepsy and a dysembryoplastic neuroepithelial tumor. During fMRI recording, EEG showed frequent F4 spikes and slow waves (red outline). She underwent a lesionectomy of the right frontal cortex (resection area bounded by green outline). At 1 year after surgery, the outcome was consistent with ILAE outcome class 1. A: The result of conventional EEG-fMRI analysis using an ER approach. The voxel of the third-highest $t$-value $(t=5.97$, MNI space $[x, y, z]=[-10,26,30]$, arrow) was outside the resection area but within $2 \mathrm{~cm}$ from the resection margin and therefore judged as "borderline." The significance level was set at $p<0.05$ corrected for multiple comparisons using an FEWp. B: The result of subsecond EEG-fMRI analysis using the SSWAS map. The voxel of the first-highest activation frequency (activation frequency $=91, \operatorname{MNI}[x, y, z]=[2,32,34]$, arrow) was inside the resection area and considered "concordant." The significance level was set at $p<0.05$ corrected for multiple comparisons using an FWEc. $R=$ right, $s=$ second. Figure is available in color online only.

identification of hemodynamic responses related to epileptic foci. ${ }^{13}$ We extended their method, using the sequence of topographic maps during IEDs from EEG data recorded inside the scanner as a reference to detect dynamic activation changes with millisecond-level temporal resolution, from which SSWAS maps could be constructed for epileptic focus detection. Unlike the study of Grouiller et al., we did not use EEG data outside the scanner as a reference. This was to avoid mismatching IED morphology and differences in spatial sampling inside and outside the scanner. In addition, we used canonical HRF, a common practice in EEG-fMRI analysis. Although the use of multiple HRFs can further increase the percentage of patients with significant BOLD responses, ${ }^{6}$ this approach may demonstrate various phenomena, including artifacts and propagated epileptic activities. ${ }^{19}$ Furthermore, all fMRI was recorded in a single continuous 15 -minute session, which is shorter than previous studies. ${ }^{4,7}$ A longer recording time can further improve the detection rate of significant BOLD responses due to the potential increase in the number of IEDs occurring within the scan period, but imposes a greater burden on patients who have to endure the restrictions on body movement and loud noise for a longer time.
The sensitivity and specificity of the SSWAS map compared with resection areas and good surgical outcomes were adequate for presurgical evaluation in focal epilepsy, comparable to existing findings. In a previous ER-based analysis study using multiple HRFs for focal epilepsy, the sensitivity and specificity were $87.5 \%$ and $76.9 \%$, respectively. ${ }^{4}$ In a recent topography-based analysis using canonical HRF for temporal lobe epilepsy (TLE), the sensitivity and specificity were $81 \%$ and $79 \%$, respectively. The primary cluster that contains the voxel with maximum t-value has also been shown to be consistent not only with the irritative zone, but also with the seizure onset zone (SOZ) in $24(77 \%)$ of 31 patients with drug-resistant focal epilepsy. ${ }^{17}$ In this study, both SOZ and significant BOLD responses using SSWAS maps were detected in 4 patients and the SOZ was concordant with the cluster containing the voxel with the highest activation frequency in 2 patients (patients 1 and 2) for whom surgical outcome was ILAE class 1. This suggests that the highest activation frequency of the BOLD response may predict the SOZ as well as the location of the maximum t-value, and contribute to adequate sensitivity and specificity of SSWAS maps. Other useful imaging modalities include magnetic source imaging, high-density electric source imaging, FDG-PET, SPECT, 


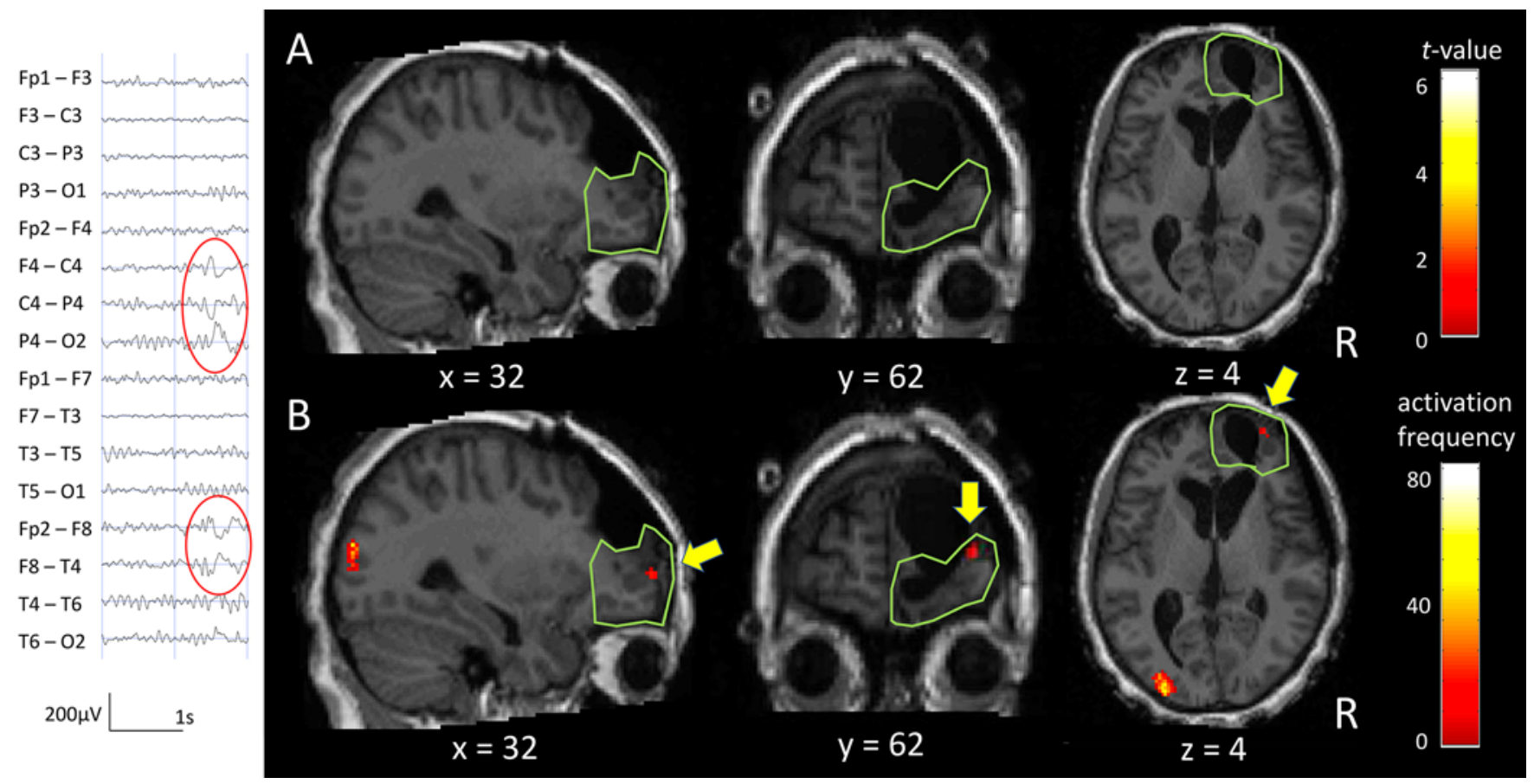

FIG. 3. Case 5. A case with no significant hemodynamic responses using conventional ER analysis and concordant hemodynamic responses using subsecond analysis. This patient was a 24 -year-old woman with right frontal lobe epilepsy and hemorrhagic scar from an arteriovenous malformation. During fMRI recording, EEG showed frequent F8/C4 spikes and slow waves (red outlines). She underwent a lesionectomy of the right orbitofrontal cortex (resection area bounded by green outline). At 1 year after surgery, the outcome was consistent with ILAE outcome class 1. A: The result of conventional EEG-fMRI analysis using an ER approach. There was no significant hemodynamic response. The significance level was set at $p<0.05$, corrected for multiple comparisons using an FWEc. B: The result of subsecond EEG-fMRI analysis using the SSWAS map. The voxel with the third-highest activation frequency (activation frequency $=19$, MNI space $[x, y, z]=[32,62,4]$, arrow) was inside the resection area and therefore considered "concordant." The significance level was set at $p<0.05$, corrected for multiple comparisons using an FWEc. Figure is available in color online only.

and ultra-high-field MRI. ${ }^{8,14,16}$ EEG-fMRI and these modalities are complementary, and combining these methods may enable precise assessment of the epileptic foci before invasive icEEG recording to improve postoperative outcomes for intractable focal epilepsy.

Subsecond analysis can help avoid human bias and minimize efforts at manual IED detection in EEG-fMRI analyses. EEG data recorded inside the scanner contain various artifacts that could lead to some true IEDs being missed and some spikelike waveforms being selected by mistake, even for experienced EEG experts interpreting the EEG recordings. ${ }^{23,26,30}$ This, in turn, could affect the statistical analysis of the simultaneously recorded fMRI data and reduce the performance of EEG-fMRI analyses, such as ER-based approaches, in localizing epileptic foci among patients with epilepsy. The method evaluated in this study avoids this problem and only requires experienced epileptologists to mark several clear IEDs for it to work. This could save large amounts of time and money in obtaining the final results and could boost EEG-fMRI analysis as a more practical neuroimaging modality for evaluating epilepsy patients. Moreover, when no spikes are visible on EEG recorded inside the scanner, the method also allows the use of EEG data recorded outside the scanner to make the reference topographic map for detect- ing latent IEDs, for which voltages are usually lower than those of artifacts. More studies are needed to validate the utility of this method under such conditions.

When we used SSWAS maps, we defined clusters containing voxels of the first- to the third-highest activation frequencies as significant. Khoo and colleagues showed that IEDs on the icEEG electrode adjacent to the voxel of the maximum t-value tended to precede IEDs on the other icEEG electrodes, and the region of the maximum t-value was consistent with the origin of IEDs, called "the spike onset zone." ${ }^{18}$ On this basis, it is ideal to define only the cluster with the voxel of highest activation frequency as significant. Under that condition, the sensitivity and specificity of SSWAS maps were $50.0 \%$ and $100.0 \%$, respectively. However, SSWAS maps can increase the candidates of significant BOLD clusters for focus detection from $36 \%$ to $91 \%$ using the sequence of topographic maps during IEDs. For that reason, it is reasonable that clusters with the voxels of the first- to third-highest activation frequency were significant when we used a subsecond approach for EEG-fMRI analysis in patients with focal epilepsy. The research task was to find how to detect the true epileptic focus from the three clusters and the epileptic network architecture spanning between them.

The findings of the present study were limited by the 
small sample size, the short follow-up period, and the fact that SOZ on icEEG was not detected in all patients. More participants, longer follow-up, and comparison with other neuroimaging modalities may lead to a better understanding of the utility of subsecond EEG-fMRI analysis methods. In addition, we believe that subsecond analysis could be generalized to other types of epilepsy. Studies using subsecond analysis in the investigation of benign epilepsy with centrotemporal spikes, epileptic encephalopathy with continuous spike waves during slow-wave sleep, and West syndrome are currently underway.

\section{Conclusions}

We have demonstrated the clinical utility of SSWAS maps as effective tools for presurgical evaluation of pharmacoresistant focal epilepsy. Our findings showed that SSWAS maps offer a higher detection rate of spike-related significant BOLD signals than conventional ER-based analysis approaches and can be predictive of surgical outcomes. This approach also reduces the burden of manual IED detection on EEG recorded inside the scanner. Taken together, these findings have provided additional evidence for the usefulness of EEG-fMRI as a more practical modality in diagnosing patients with epilepsy that could potentially reduce the necessity for invasive icEEG recordings.

\section{Acknowledgments}

This research was supported by The Japan Epilepsy Research Foundation (JERF) with the project title "A study for focus detection by novel techniques with resting-state fMRI, EEG-fMRI and MEG, and surgical outcomes in epilepsy surgery" (principal investigator Satoshi Maesawa) and grants from the Japan Agency for Medical Research and Development (AMED) under grant no. JP18dm0207005. The funders had no role in the study design, data collection, analysis, and interpretation of the data, or preparation of the manuscript. We would also like to acknowledge Mr. Akira Ishizuka (radiological technologist) for his technical support during MRI scans. Jun Natsume belongs to the Department of Developmental Disability Medicine in the Nagoya University Graduate School of Medicine, which is the laboratory endowed by Aichi Prefecture.

\section{References}

1. Aghakhani Y, Kobayashi E, Bagshaw AP, Hawco C, Bénar CG, Dubeau F, et al: Cortical and thalamic fMRI responses in partial epilepsy with focal and bilateral synchronous spikes. Clin Neurophysiol 117:177-191, 2006

2. Al-Asmi A, Bénar CG, Gross DW, Khani YA, Andermann F, Pike B, et al: fMRI activation in continuous and spiketriggered EEG-fMRI studies of epileptic spikes. Epilepsia 44:1328-1339, 2003

3. Allen PJ, Josephs O, Turner R: A method for removing imaging artifact from continuous EEG recorded during functional MRI. Neuroimage 12:230-239, 2000

4. An D, Fahoum F, Hall J, Olivier A, Gotman J, Dubeau F: Electroencephalography/functional magnetic resonance imaging responses help predict surgical outcome in focal epilepsy. Epilepsia 54:2184-2194, 2013

5. Bagarinao E, Maesawa S, Ito Y, Usui N, Natsume J, Watanabe $\mathrm{H}$, et al: Detecting sub-second changes in brain activation patterns during interictal epileptic spike using simultaneous EEG-fMRI. Clin Neurophysiol 129:377-389, 2018
6. Bagshaw AP, Aghakhani Y, Bénar CG, Kobayashi E, Hawco C, Dubeau F, et al: EEG-fMRI of focal epileptic spikes: analysis with multiple haemodynamic functions and comparison with gadolinium-enhanced MR angiograms. Hum Brain Mapp 22:179-192, 2004

7. Coan AC, Chaudhary UJ, Grouiller F, Campos BM, Perani S, De Ciantis A, et al: EEG-fMRI in the presurgical evaluation of temporal lobe epilepsy. J Neurol Neurosurg Psychiatry 87:642-649, 2016

8. De Ciantis A, Barba C, Tassi L, Cosottini M, Tosetti M, Costagli M, et al: 7T MRI in focal epilepsy with unrevealing conventional field strength imaging. Epilepsia 57:445-454, 2016

9. Engel J Jr: Surgery for seizures. N Engl J Med 334:647-652, 1996

10. Flanagan D, Abbott DF, Jackson GD: How wrong can we be? The effect of inaccurate mark-up of EEG/fMRI studies in epilepsy. Clin Neurophysiol 120:1637-1647, 2009

11. Gotman J: Epileptic networks studied with EEG-fMRI. Epilepsia 49 (Suppl 3):42-51, 2008

12. Gotman J, Pittau F: Combining EEG and fMRI in the study of epileptic discharges. Epilepsia 52 (Suppl 4):38-42, 2011

13. Grouiller F, Thornton RC, Groening K, Spinelli L, Duncan JS, Schaller K, et al: With or without spikes: localization of focal epileptic activity by simultaneous electroencephalography and functional magnetic resonance imaging. Brain 134:2867-2886, 2011

14. Guerrini R, Duchowny M, Jayakar P, Krsek P, Kahane P, Tassi L, et al: Diagnostic methods and treatment options for focal cortical dysplasia. Epilepsia 56:1669-1686, 2015

15. Hamer HM, Morris HH, Mascha EJ, Karafa MT, Bingaman WE, Bej MD, et al: Complications of invasive video-EEG monitoring with subdural grid electrodes. Neurology 58:97103,2002

16. Heers M, Hedrich T, An D, Dubeau F, Gotman J, Grova C, et al: Spatial correlation of hemodynamic changes related to interictal epileptic discharges with electric and magnetic source imaging. Hum Brain Mapp 35:4396-4414, 2014

17. Khoo HM, Hao Y, von Ellenrieder N, Zazubovits N, Hall J, Olivier A, et al: The hemodynamic response to interictal epileptic discharges localizes the seizure-onset zone. Epilepsia 58:811-823, 2017

18. Khoo HM, von Ellenrieder N, Zazubovits N, He D, Dubeau F, Gotman J: The spike onset zone: The region where epileptic spikes start and from where they propagate. Neurology 91:e666-e674, 2018

19. Lemieux L, Laufs H, Carmichael D, Paul JS, Walker MC, Duncan JS: Noncanonical spike-related BOLD responses in focal epilepsy. Hum Brain Mapp 29:329-345, 2008

20. Mandelkow H, Halder P, Boesiger P, Brandeis D: Synchronization facilitates removal of MRI artefacts from concurrent EEG recordings and increases usable bandwidth. Neuroimage 32:1120-1126, 2006

21. Niazy RK, Beckmann CF, Iannetti GD, Brady JM, Smith SM: Removal of FMRI environment artifacts from EEG data using optimal basis sets. Neuroimage 28:720-737, 2005

22. Ogawa S, Lee TM, Kay AR, Tank DW: Brain magnetic resonance imaging with contrast dependent on blood oxygenation. Proc Natl Acad Sci U S A 87:9868-9872, 1990

23. Omidvarnia A, Kowalczyk MA, Pedersen M, Jackson GD: Towards fast and reliable simultaneous EEG-fMRI analysis of epilepsy with automatic spike detection. Clin Neurophysiol 130:368-378, 2019

24. Rosenow F, Lüders H: Presurgical evaluation of epilepsy. Brain 124:1683-1700, 2001

25. Salek-Haddadi A, Diehl B, Hamandi K, Merschhemke M, Liston A, Friston K, et al: Hemodynamic correlates of epileptiform discharges: an EEG-fMRI study of 63 patients with focal epilepsy. Brain Res 1088:148-166, 2006 
26. Scheuer ML, Bagic A, Wilson SB: Spike detection: Interreader agreement and a statistical Turing test on a large data set. Clin Neurophysiol 128:243-250, 2017

27. Schuele SU, Lüders HO: Intractable epilepsy: management and therapeutic alternatives. Lancet Neurol 7:514-524, 2008

28. Thornton R, Laufs H, Rodionov R, Cannadathu S, Carmichael DW, Vulliemoz S, et al: EEG correlated functional MRI and postoperative outcome in focal epilepsy. J Neurol Neurosurg Psychiatry 81:922-927, 2010

29. Thornton R, Vulliemoz S, Rodionov R, Carmichael DW, Chaudhary UJ, Diehl B, et al: Epileptic networks in focal cortical dysplasia revealed using electroencephalographyfunctional magnetic resonance imaging. Ann Neurol 70:822-837, 2011

30. Tousseyn S, Dupont P, Robben D, Goffin K, Sunaert S, Van Paesschen W: A reliable and time-saving semiautomatic spike-template-based analysis of interictal EEG-fMRI. Epilepsia 55:2048-2058, 2014

31. Waites AB, Shaw ME, Briellmann RS, Labate A, Abbott DF, Jackson GD: How reliable are fMRI-EEG studies of epilepsy? A nonparametric approach to analysis validation and optimization. Neuroimage 24:192-199, 2005

32. Wiebe $\mathrm{S}$, Jette N: Pharmacoresistance and the role of surgery in difficult to treat epilepsy. Nat Rev Neurol 8:669-677, 2012

33. Wieser HG, Blume WT, Fish D, Goldensohn E, Hufnagel A, King D, et al: ILAE Commission Report. Proposal for a new classification of outcome with respect to epileptic seizures following epilepsy surgery. Epilepsia 42:282-286, 2001

\section{Disclosures}

The authors report no conflict of interest concerning the materials or methods used in this study or the findings specified in this paper.

\section{Author Contributions}

Conception and design: Ito, Maesawa. Acquisition of data: Ito, Maesawa, Okai, Nakatsubo. Analysis and interpretation of data: Ito, Maesawa, Bagarinao, Okai. Drafting the article: Ito. Critically revising the article: Maesawa, Bagarinao. Reviewed submitted version of manuscript: Yamamoto, Kidokoro, Usui, Natsume, Hoshiyama, Wakabayashi, Sobue, Ozaki. Administrative/technical/material support: Yamamoto, Kidokoro, Usui, Natsume, Hoshiyama, Wakabayashi, Sobue, Ozaki.

\section{Correspondence}

Yuji Ito: Brain \& Mind Research Center, Nagoya University, Aichi, Japan.yuji.ito@med.nagoya-u.ac.jp. 